\title{
A Machine Learning Model of Cultural Change: Role of Prosociality, Political Attitudes, and Protestant Work Ethic
}

\author{
Abhishek Sheetal and Krishna Savani \\ Nanyang Technological University
}

April 26, 2021

(C) 2021, American Psychological Association. This paper is not the copy of record and may not exactly replicate the final, authoritative version of the article. Please do not copy or cite without authors' permission. The final article will be available, upon publication, via its DOI: 10.1037/amp0000868 
Abstract. What attitudes, values, and beliefs serve as key markers of cultural change? To answer this question, we examined 221,485 respondents from the World Values Survey, a multi-wave cross-country survey of people's attitudes, values and beliefs. We trained a machine learning model to classify respondents into seven waves (i.e., periods). Once trained, the machine learning model identified a separate group of 24,611 respondents' wave with a balanced accuracy of $77 \%$. We then queried the model to identify the attitudes, values, and beliefs that contributed the most to its classification decisions, and therefore, served as markers of cultural change. These included religiosity, social attitudes, political attitudes, independence, life satisfaction, Protestant work ethic, and prosociality. Although past research in cultural change has discussed decreasing religiosity and increasing liberalism and independence, it has not yet identified Protestant work ethic, political orientation, and prosociality as values relevant to cultural change. Thus, the current research points to new directions for future research on cultural change that might not be evident from either a deductive or an inductive approach. This research illustrates that the abductive approach of machine learning, which focuses on the most likely explanations for an outcome, can provide novel insights.

Public Significance Statement. This research found that in recent years, people around the world have been becoming less religious and more liberal in their social attitudes and political orientation. People have been valuing independence more, although there appears to be a decline in independence in the last few years. The extent to which people emphasize hard work, thrift, and acting in a prosocial manner has also declined in recent years.

Keywords: cultural change; gradient boosting; machine learning; World Values Survey 


\section{A Machine Learning Model of Cultural Change: Role of Political Attitudes, Protestant}

\section{Work Ethic, and Prosociality}

Recent research in psychology has made substantial progress at documenting and predicting cultural change (Varnum \& Grossmann, 2017). An emerging conclusion from this research is that cultural practices have been moving toward greater individualism over time. For example, the use of first-person pronouns has been increasing over time (Twenge et al., 2013), as has been the general use of words related to independence and individualism (Greenfield, 2013; Grossmann \& Varnum, 2015; Twenge et al., 2012). These shifts can be traced to ecological changes, such as decreases in pathogen prevalence (Varnum \& Grossmann, 2016), and to sociostructural shifts, such as those from rural to urban societies, agriculture to commerce, joint to nuclear households, and toward fewer children, more education, and more wealth (Greenfield, 2016; Grossmann \& Varnum, 2015; Santos et al., 2017).

Much of the past research on cultural change has focused on sociostructural factors, probably because these variables are readily available over long periods and across many countries. Some research has utilized multi-country multi-wave surveys to document the pattern of cultural change in people's attitudes and values. For example, researchers have used the World Values Survey (WVS; Haerpfer et al., 2020) to argue that people in most countries are moving toward greater individualism, i.e., emphasizing self-expression, emphasizing friends over family members, and emphasizing the value of independence in children (Grossmann \& Varnum, 2015; Hamamura, 2012; Inglehart \& Baker, 2000; Santos et al., 2017). Many cultures are also moving toward a greater emphasis on modern, postindustrial, secular-rational values rather than traditional values; however, this shift is not as stark as the increase in selfexpression (Inglehart \& Baker, 2000).

Although it has been very productive, nearly all past research on cultural changes in attitudes, values, and beliefs suffer from a few limitations. First, out of the dozens of attitudes, values, and beliefs assessed in large multi-country multi-wave surveys (e.g., the WVS), 
researchers typically focused on a select few questions (e.g., four questions examined by Hamamura, 2012, or three questions analyzed by Santos et al., 2017). Past research has primarily focused on changes in individualism (Greenfield, 2013; Grossmann \& Varnum, 2015; Hamamura, 2012; Inglehart \& Baker, 2000; Santos et al., 2017; Twenge et al., 2012). Although it is possible that the studied values are indeed the most important cultural dimensions that are changing over time, it is also possible that other attitudes, values, and beliefs might be similarly strong markers of cultural change.

Second, researchers have typically assumed that past patterns of cultural change would persist into the future, but cultural change can sometimes be nonmonotonic. For example, divorce rates in the US peaked around 1980 and have since been declining (Hamamura, 2012). More generally, traditional statistical analyses require researchers to specify how they expect a given variable to change over time (e.g., linearly), but these assumptions might not hold in many cases given the complexity of cultural change (Erceg-Hurn \& Mirosevich, 2008). Third, past research has rarely provided an independent test of the predictions of a statistical model of cultural change (see Varnum \& Grossmann, this issue, for an exception). Thus, it is possible that any given statistical model has overfit the available data and is unlikely to predict future data with similarly high accuracy.

In the current research, we seek to address these shortcomings by building a machine learning model of cultural change. First, unlike traditional analyses, machine learning models can handle a large number of variables, thereby allowing us to use all attitudes, values, and beliefs that were measured in a multi-country, multi-wave survey. Thus, we can objectively identify attitudes, values, and beliefs that serve as markers of cultural change from all the attitudes, values, and beliefs consistently measured in the survey. Second, unlike traditional regression-based analyses, machine learning models do not assume any functional form. Thus, they can model non-linear changes in attitudes, values, and beliefs over time. Third, the accuracy of the machine learning model's predictions is tested in a subset of the data to which 
the model was never exposed (i.e., the unseen data), thereby providing a metric of accuracy that is not susceptible to overfitting.

\section{Method}

All code is available at the online data repository for this project: https://osf.io/9yx8s (see file Code.zip). The procedure that we used is illustrated in Figure 1.

\section{Dataset}

We decided to use the World Values Survey dataset (Haerpfer et al., 2020) because it is one of the most comprehensive international social science survey datasets currently available. It contains responses of 423,948 people from 104 different countries. The cross-sectional survey was conducted over seven periods (called waves): 1981-1984, 1989-1993, 1994-1998, 1999-2004, 2005-2009, 2010-2014, and 2017-2019. Thus, this dataset allows us to examine how people's attitudes, values, and beliefs have been changing over time in different parts of the world. Past research on cultural change has used this dataset (Hamamura, 2012; Inglehart \& Baker, 2000; Santos et al., 2017). The WVS waves are highly irregular-the number of years covered by each wave and the gap between successive waves varies quite a bit. Thus, we treated each wave as a category rather than treating all waves as falling on a continuum.

The machine learning model that we used can automatically handle missing values. However, to minimize the proportion of missing values in the data, we only included 53 attitudes, values, and beliefs (cultural variables in short) that were asked in all seven waves in at least one country. A question assessing religious denomination (variable f025) included over 100 categorical response options, so we re-coded it into a binary variable $(0=$ no religious denomination, 1 = some religious denomination). Many of these 53 questions were not asked in several countries in a given wave. Once again, to minimize the proportion of missing data, we only included country-wave combinations in which all 53 cultural questions were administered. 


\section{Figure 1}

Illustration of the machine learning procedure.

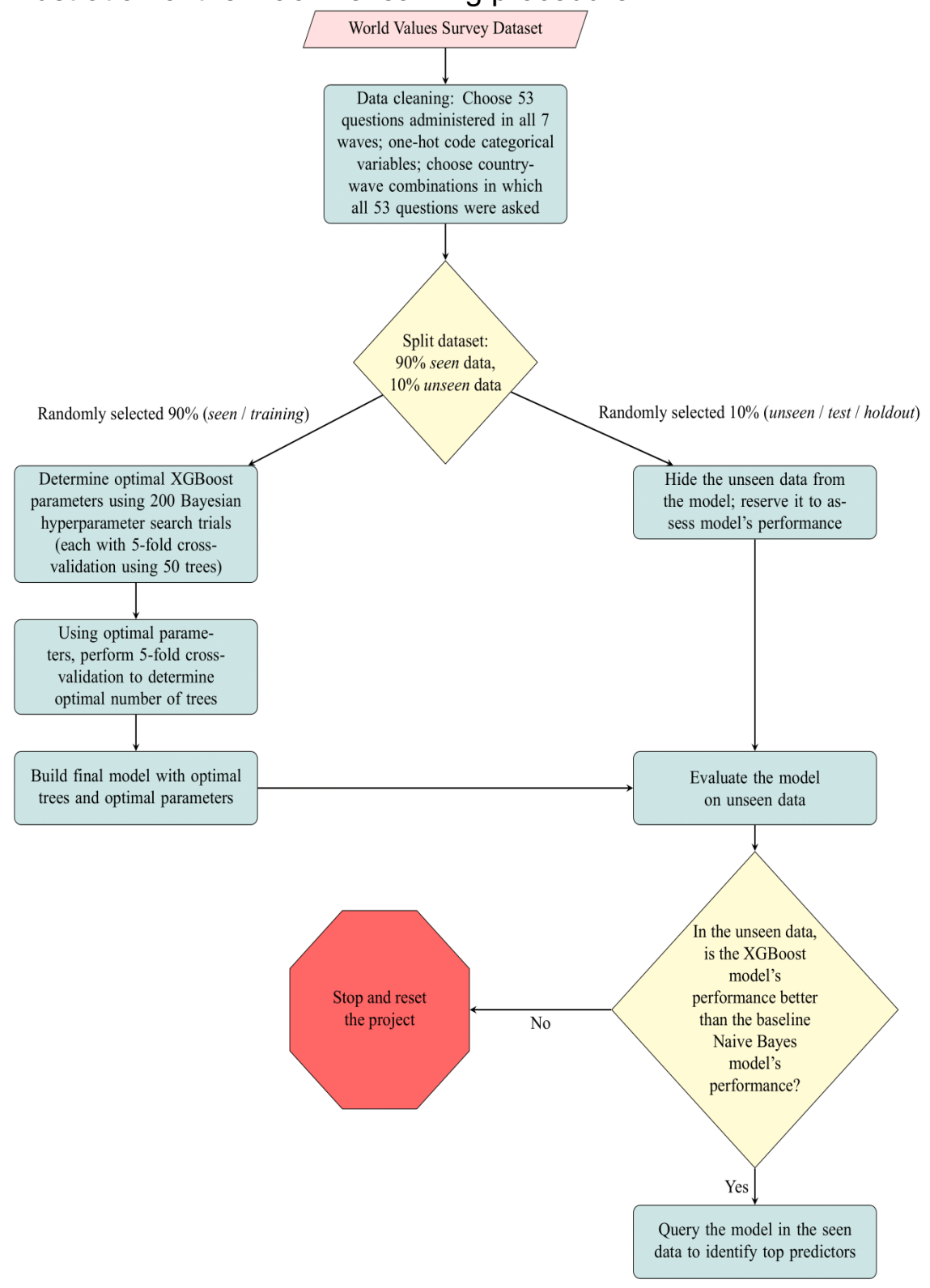

This procedure resulted in a dataset with 246,096 respondents from 83 countries. Overall, $3.00 \%$ of the values were missing in this dataset. The list of variables and country-wave combinations included is available in the online data repository. The categorical variables e003, e004, and f034 were one-hot coded (i.e., a new variable was created for each response option), which resulted in a total of 61 variables. A list of all variables included is uploaded on the online data repository (see file WVS_variables_used.xlsx). A list of all country-subregion-wave 
combinations included in our dataset is also uploaded in the online repository (see file Country_wave_combinations.xlsx).

Only one country (Mexico) was included in all seven waves; one (Argentina) in six waves; three (Brazil, Chile, and the US) in five waves; eight in four waves; 12 in three waves; 25 in two waves; and 33 in only one wave. Thus, for the vast majority of the countries, there was insufficient data to model how individuals' attitudes, values, and beliefs changed across different waves. Therefore, we combined data from multiple countries within each ISO sub-region (ISO, 2006). There was data from Sub-Saharan Africa and Latin America and the Caribbean for all seven waves; Eastern Asia, Southern Asia, and Southern Europe for six waves; Northern America, South-eastern Asia, and Eastern Europe for five waves; Western Asia, North Europe, and Australia and New Zealand for four waves; Northern Africa for two waves; and Central Asia for only one wave. Following past research (Inglehart \& Baker, 2000; Schwartz, 2006), we assumed that countries in a given sub-region are culturally similar. We did so because countries within a given region typically experience a similar ecology (Oishi \& Graham, 2010), and because national boundaries are often a result of historical accidents (e.g., arbitrary colonial boundaries; Englebert et al., 2002). National boundaries are dichotomous, but cultural variation over geographical regions is more of a gradient (Cohen, 2001).

We used the holdout technique to test the model's predictions. That is, we randomly selected 24,611 respondents (10\% of the data) and designated them as the unseen sample. The remaining 221,485 respondents were used to build the machine learning model (i.e., the training data). The machine learning model was not exposed to the unseen sample at any stage until it was finalized. Thus, the model's accuracy was assessed on these "new respondents" that the model had never seen previously. This process ensured that the training data and the unseen data were separated in all steps of the modeling process.

\section{Building the machine learning model}

Our dataset was large enough that it would take weeks for it to be analyzed using the 
original CPU-based random forest machine learning method (Breiman, 2001) to complete the analysis on a 128-thread CPU. We thus performed the model training on the computer's graphics card (Nvidia GTX 1070) to reduce the model building time (Strigl et al., 2010). We used the XGBoost (Chen \& Guestrin, 2016) package in $R$, which implements gradient boosted decision trees. The base random forest model is a special case of the more generic XGBoost model (XGBoost Developers, 2020a). XGBoost has been used in past research in psychology (e.g., Goretzko \& Buhner, 2020) and economics (e.g., Kleinberg et al., 2018). Unlike random forest, XGBoost automatically handles missing values during the process of building a model; that is, it creates parallel paths for each possible missing value (e.g., assuming that a missing binary variable had a value of 0 in one path and a value of 1 in another path), and learns the optimal default path over successive iterations (Chen \& Guestrin, 2016). In contrast, a standard random forest model would drop all observations with even a single missing value (Breiman, 2001).

We built three different models using the training data. In model 1, we ignored the country and sub-region that each participant belonged to, and instead focused on the wave in which each participant was sampled. The model thus learned to classify each participant in one of seven classes, representing the seven waves. In model 2, we used a hierarchical structure with waves nested within sub-regions. This more complex model learned to classify each participant's wave along with their sub-region (66 classes representing all wave-subregion pairs in our dataset). In model 3, we treated waves as nested within countries. Thus, the model learned to classify each participant's wave and their country (179 classes representing all wavecountry pairs in our dataset). As all models classified participants into one of many classes, we used the multiclass-log-loss loss function (also known as the categorical cross-entropy loss function; Zhang \& Sabuncu, 2018).

Models trained to classify observations often accurately classify observations from the majority class but perform poorly when classifying observations from minority classes (Guo et 
al., 2008). As there was an uneven number of respondents in each wave (model 1), each wavesubregion combination (model 2), and each wave-country combination (model 3), we assigned class weights to each observation. The class weights variable required by XGBoost must be a vector of $N$ values, where $N$ is the number of classes in the model (e.g., a vector of size 7 in model 1). For each respondent in the training data, we divided the proportion of respondents belonging to the least represented class in the training data by the proportion of respondents belonging to the class to which this respondent belonged. Class weights were not used when the model was tested on the unseen sample. Note that class weights are distinct from the participant weights that the WVS provides (variable s017), which indicate whether each respondent's demographics characteristics are overrepresented or underrepresented in the survey relative to the population. It is not possible to simultaneously use class weights and participant weights in XGBoost.

XGBoost uses a three-step process to build the model. The first two steps identify the optimal model parameters, and the third step builds the actual machine learning model. In the first step, instead of using the default parameters in the XGBoost package, we conducted a hyperparameter search to identify a relatively superior set of XGBoost parameters (see Figure 2 for an illustration of the procedure). There are three methods for hyperparameter search. The first method is random search, in which the algorithm randomly draws a value of each parameter from the provided range across several iterations and picks the parameter set that generated the lowest loss value. The second method is grid search, in which the parameter values are stepped in increments over the parameter range until the entire space is exhausted. The third is Bayesian search, which uses information about how the loss value changed across all previous parameter combinations to guess the next set of parameters. We conducted a hyperparameter optimization search using the BayesOpt library (Martinez-Cantin, 2014), which can be applied to any parameter optimization problem. BayesOpt used 5-fold cross-validation in each iteration; that is, $80 \%$ of the data was designated as the training data, which was used to 
identify a set of parameters, and the remaining $20 \%$ of the data was designated as the validation data, which was used to test the parameters. Across 200 iterations, the function experimented with various combinations of seven model parameters to identify the optimal set of parameters that generates the lowest multi-class log-loss in the validation data. Several other parameters were fixed. See Table S1 in the Supplementary Materials for details about the parameters.

\section{Figure 2}

Illustration of the Bayesian hyperparameter search procedure.

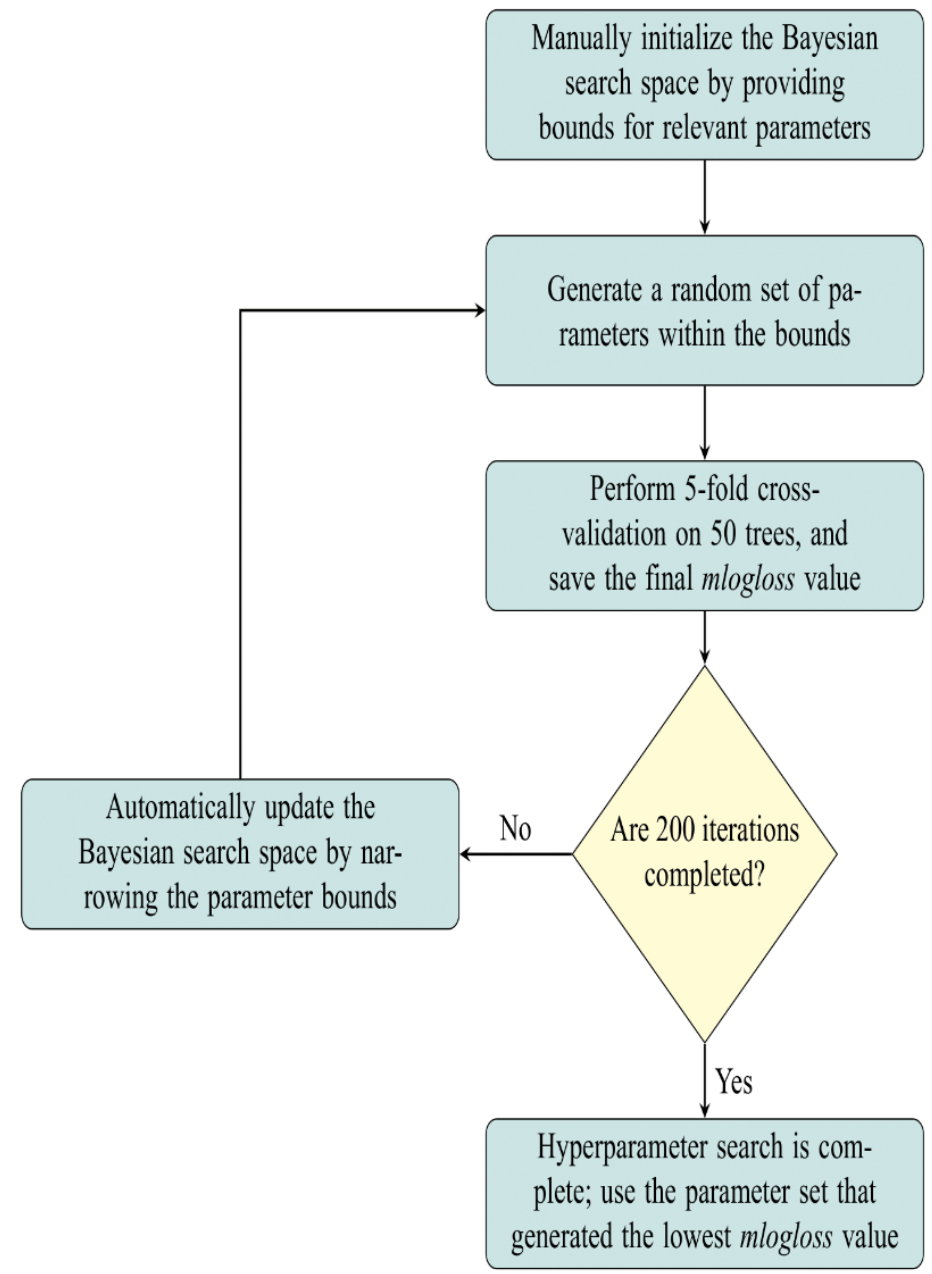

In the second step, we took the optimal hyperparameter values determined in the first step and conducted a second parameter search procedure to determine the optimal number of trees that would minimize the loss function. Once again, we used 5-fold cross-validation. The 
model started with one tree and assessed the loss value in the validation data. The model then kept increasing the number of trees across successive iterations up to a maximum of 2000 trees. We set early stopping at 5 , which meant that if the validation data's loss value did not decrease appreciably after five iterations, the most recent number of trees was selected as the optimal parameter. In the third step, we took the parameters identified in the first two steps and built the final model. We repeated each of these three steps for each of our three models.

\section{Results}

\section{Accuracy in classifying participants in the unseen sample}

Once each model was finalized, we tested its performance in the unseen sample. Note that the model was never exposed to the unseen sample, and thus, the unseen sample serves as a set of "new participants" that the model has to classify based on each participants' attitudes, values, and beliefs. Table 1 reports the summary accuracy statistics.

Table 1

Accuracy statistics of the three XGBoost models in the unseen sample.

\begin{tabular}{lccc}
\hline \multicolumn{1}{c}{ Statistic } & Model 1 & Model 2 & Model 3 \\
\hline $\begin{array}{l}\text { No information } \\
\text { accuracy }\end{array}$ & $23.7 \%$ & $5.7 \%$ & $1.43 \%$ \\
Naïve Bayes & $29.87 \%[29.30$, & $13.30 \%[12.88$, & $15.53 \%[15.08$, \\
accuracy & $30.45]$ & $13.73]$ & $15.98]$ \\
\hline XGBoost accuracy & $59.18 \%[58.56$, & $51.53 \%[50.90$, & $50.49 \%[49.87$, \\
Kappa & $59.80]$ & $52.16]$ & $51.12]$ \\
Area under the & $50.55 \%$ & $50.22 \%$ & $50.18 \%$ \\
curve (AUC) & $90.29 \%$ & $96.54 \%$ & $97.54 \%$ \\
$\begin{array}{l}\text { Average sensitivity } \\
\text { I recall }\end{array}$ & & & \\
Average specificity & $61.89 \%$ & $51.56 \%$ & $49.15 \%$ \\
$\begin{array}{l}\text { Average precision } \\
\text { Average F1 }\end{array}$ & & & \\
Average balanced & $92.96 \%$ & $99.24 \%$ & $99.72 \%$ \\
accuracy & $58.37 \%$ & $55.96 \%$ & $48.68 \%$ \\
\hline
\end{tabular}

The first five accuracy metrics reported in Table 1 are applicable to the model as a whole. The no information accuracy refers to the baseline accuracy rate if the model predicted that all participants in the unseen sample belonged to the most common class (e.g., wave 7 in model 1) In other words, if a rogue model 1 predicted that every observation belonged to the 
majority class (i.e., wave 7 ), then it's accuracy would be $23.7 \%$. The Naïve Bayes accuracy provides yet another benchmark; this model used Bayes' theorem to predict participants' class based on their attitudes, values, and beliefs, with the assumption that each of these variables independently contributes to the prediction (i.e., that there are no interactions; Rish, 2001). This accuracy is in the $18-28 \%$ range across the three models. The XGBoost model's accuracy ranged from $50-60 \%$, which was substantially higher than the two benchmarks. Cohen's kappa refers to the extent to which the model's accuracy is higher than the no information accuracy; our kappa values of around $50 \%$ would be categorized as moderate according to Landis and Koch (1977). The XGBoost model's area under the curve (AUC) was over $90 \%$ for all three models. The AUC refers to the probability that when presented with two randomly selected participants from different classes (e.g., participant \#1 from wave 1 and participant \#2 from wave 2), the model predicts that participant \#1's chances of being from wave 1 are higher than participant \#2's chances, and vice-versa for wave 2. For multi-class classifiers, as in the present case, the AUC is a more relevant metric than raw accuracy (Huang \& Ling, 2005).

The remaining accuracy metrics are specific to each class. The detailed class-by-class statistics for all three models are uploaded on the online data repository (see file Accuracy_statistics.xlsx). The average statistic across all classes is reported in Table 1. The models' sensitivity or recall, which refers to the proportion of participants from a given class who were accurately classified as belonging to that class, indicates that about half the participants belonging to a given class were accurately classified as such. The models' specificity, which refers to the proportion of participants not belonging to a given class who were accurately classified as not belonging to that class, is very high, exceeding $90 \%$. The models' precision, which refers to the proportion of participants who actually belonged to the predicted class overall participants classified as belonging to that class, was above just about $50 \%$ across the three models. The models' F1 statistic, which is the harmonic mean of precision and recall, was again about $50 \%$. The models' balanced accuracy, which is the average of sensitivity and 
specificity, was between $74-77 \%$, well above the raw accuracy rate of $50-59 \%$. The balanced accuracy is particularly relevant for imbalanced data, in which some classes are overrepresented, and others are under-represented, as in the present case.

We submit that the moderate raw accuracy rate of $50-59 \%$ is a function of the number of classes that the model was trained to distinguish (7 in model 1, 66 in model 2, and 179 in model 3). With fewer classes, the model's accuracy should improve. To illustrate, we trained another XGBoost model to distinguish respondents from just waves 6 and 7 , which resulted in a binary classification problem. This model's accuracy was $81.56 \%(95 \% \mathrm{Cl}[80.71,82.40])$, significantly higher than model 1's accuracy in the 7-class classification problem.

\section{Querying the model}

XGBoost includes an in-built feature importance method (XGBoost Developers, 2020b) that can be used to rank order the predictor variables in terms of their contribution to the model's classification (often referred to as gain or impurity or gini; see Breiman et al., 1984). Alternatively, it is possible to use a permutation-based method, which would shuffle the values 'of each predictor variable one at a time and assess the extent to which the model's loss value changed as a consequence (Altmann et al., 2010). Variables whose perturbations lead to a bigger change in the model's loss value are relatively more important predictors. We decided to use a permutation-based method because "Permutation-based importance methods are a reliable technique that does not suffer from the bias existing in Gini impurity which might inflate the importance of continuous and high-cardinality categorical variables" (Gomez-Ramirez et al., 2020, p. 4). Specifically, we used the model-agnostic permutation-based DALEX package

(Biecek, 2018). Note that XGBoost automatically models both linear and non-linear relationships, along with any number of complex interactions. Thus, even if the mean value of a variable stayed largely constant across waves, the variable could show up as a top predictor in the variable importance analysis if it interacted with other variables.

Table 2 presents the top predictors from the first model that was trained to classify which 
of seven waves participants were sampled in. The rankings of all 61 predictor variables (after one-hot coding) in terms of their contribution to distinguishing respondents across the seven waves globally (model 1), within each sub-region (model 2), and within each country (model 3) are uploaded on the online data repository (see file Top_Predictors_DALEX.xIsx). We discuss the top 10 variables that contributed the most to distinguishing respondents from all seven waves, as well as across successive waves, from model 1 (see file Top_predictors_model1.xlsx). For comparison, we also rank-ordered the predictors based on XGBoost's in-built feature importance algorithm and found substantial overlap with the DALEXbased feature importance rankings (see Top_predictors_XGBoost_Gini.xlsx on OSF).

To illustrate the pattern of changes in the top predictors across the seven waves in Figures 3 to 7, we ran a hierarchical linear model (HLM) for each variable featured in Table 2 using the xtreg command in STATA ${ }^{\mathrm{TM}}$. Specifically, in the original WVS dataset, we regressed each predictor on six dummy variables indicating waves 2 to 7 , respectively (wave 1 was treated as the reference category). We treated participants as nested within countries, thereby accounting for country effects. Given the paucity of waves within a number of countries, we used fixed slopes. As we accounted for country effects, the mean levels of the variables depicted in the graphs are not interpretable-only the trends over time are interpretable. The HLM code and results are uploaded on the online data repository (HLM_code_figures.pdf). As different variables were measured on different response scales, for ease of interpretation, in the graphs, we scaled all variables to range from 0 to 1 . We reverse-scored variables as needed for ease of interpretation. The HLM model was run on the original variables provided in the WVS dataset (before scaling and reverse-scoring).

Religiosity. The most important set of predictors that helped distinguish respondents across all seven waves was associated with religiosity (variables f028, f063, f025, and a040). 
Table 2. Top 10 attitudes, values, and beliefs that helped distinguish respondents from all sever waves (model 1 ), as well as those that helped distinguish respondents from each successive pairs of waves (also from model 1 ).

\begin{tabular}{|c|c|c|c|c|c|c|c|}
\hline Rank & All seven waves & $\begin{array}{c}\text { Waves } 1 \text { vs. } 2 \\
\text { (1981-1984 vs. } \\
\text { 1989-1993) } \\
\end{array}$ & $\begin{array}{c}\text { Waves } 2 \text { vs. } 3 \\
\text { (1989-1993 vs. } \\
1994-1998) \\
\end{array}$ & $\begin{array}{c}\text { Waves } 3 \text { vs. } 4 \\
(1994-1998 \text { vs. } \\
1999-2004) \\
\end{array}$ & $\begin{array}{c}\text { Waves } 4 \text { vs. } 5 \\
\text { (1999-2004 vs. } \\
2005-2009) \\
\end{array}$ & $\begin{array}{c}\text { Waves } 5-6 \\
(2005-2009 \text { vs. } \\
2010-2014) \\
\end{array}$ & $\begin{array}{c}\text { Waves } 6 \text { vs. } 7 \\
\text { (2010-2014 vs. } \\
2017-2019) \\
\end{array}$ \\
\hline 1 & $\begin{array}{l}\text { Attend religious } \\
\text { services }(0.23)\end{array}$ & $\begin{array}{l}\text { Attend religious } \\
\text { services }(0.654)\end{array}$ & $\begin{array}{l}\text { Attend religious } \\
\text { services }(0.287)\end{array}$ & $\begin{array}{l}\text { Attend religious } \\
\text { services }(0.182)\end{array}$ & $\begin{array}{l}\text { Important in } \\
\text { children: } \\
\text { independence } \\
(0.168)\end{array}$ & $\begin{array}{l}\text { Justifiability of } \\
\text { abortion }(0.179)\end{array}$ & $\begin{array}{l}\text { Justifiability of } \\
\text { abortion }(0.306)\end{array}$ \\
\hline 2 & $\begin{array}{c}\text { Justifiability of } \\
\text { homosexuality } \\
(0.198)\end{array}$ & $\begin{array}{c}\text { Important in } \\
\text { children: hard work } \\
(0.408)\end{array}$ & $\begin{array}{c}\text { Importance of God } \\
(0.145)\end{array}$ & $\begin{array}{l}\text { Importance of God } \\
(0.135)\end{array}$ & $\begin{array}{l}\text { Attend religious } \\
\text { services }(0.156)\end{array}$ & $\begin{array}{c}\text { Justifiability of } \\
\text { homosexuality } \\
(0.178)\end{array}$ & $\begin{array}{c}\text { Justifiability of } \\
\text { homosexuality } \\
(0.245)\end{array}$ \\
\hline 3 & $\begin{array}{l}\text { Justifiability of } \\
\text { abortion }(0.181)\end{array}$ & $\begin{array}{c}\text { Justifiability of } \\
\text { homosexuality } \\
(0.36)\end{array}$ & $\begin{array}{l}\text { Greater respect for } \\
\text { authority in future: } \\
\text { good or bad? } \\
(0.122)\end{array}$ & $\begin{array}{c}\text { Important in } \\
\text { children: obedience } \\
(0.101)\end{array}$ & $\begin{array}{l}\text { Important in } \\
\text { children: thrift } \\
(0.144)\end{array}$ & $\begin{array}{l}\text { Important in } \\
\text { children: } \\
\text { independence } \\
(0.157)\end{array}$ & $\begin{array}{c}\text { Importance of God } \\
(0.185)\end{array}$ \\
\hline 4 & $\begin{array}{c}\text { Importance of God } \\
(0.169)\end{array}$ & $\begin{array}{l}\text { Political orientation } \\
\qquad(0.322)\end{array}$ & $\begin{array}{l}\text { Political orientation } \\
(0.109)\end{array}$ & $\begin{array}{l}\text { Important in } \\
\text { children: } \\
\text { independence } \\
(0.095)\end{array}$ & $\begin{array}{l}\text { Important in } \\
\text { children: religious } \\
\text { faith }(0.142)\end{array}$ & $\begin{array}{l}\text { Attend religious } \\
\text { services }(0.147)\end{array}$ & $\begin{array}{c}\text { Justifiability of } \\
\text { accepting bribes } \\
(0.179)\end{array}$ \\
\hline 5 & $\begin{array}{l}\text { Important in } \\
\text { children: } \\
\text { independence } \\
(0.134)\end{array}$ & $\begin{array}{l}\text { Important in } \\
\text { children: } \\
\text { independence } \\
(0.295)\end{array}$ & $\begin{array}{c}\text { Justifiability of } \\
\text { homosexuality } \\
(0.101)\end{array}$ & $\begin{array}{l}\text { Political orientation } \\
(0.088)\end{array}$ & $\begin{array}{c}\text { Important in } \\
\text { children: obedience } \\
(0.141)\end{array}$ & $\begin{array}{l}\text { Importance of God } \\
\qquad(0.135)\end{array}$ & $\begin{array}{c}\text { Confidence in the } \\
\text { armed forces } \\
(0.178)\end{array}$ \\
\hline 6 & $\begin{array}{c}\text { Important in } \\
\text { children: hard work } \\
(0.133)\end{array}$ & $\begin{array}{c}\text { Religious } \\
\text { denomination } \\
(0.287)\end{array}$ & $\begin{array}{l}\text { Less importance on } \\
\text { work in future: good } \\
\text { or bad? }(0.099)\end{array}$ & $\begin{array}{l}\text { Greater respect for } \\
\text { authority in future: } \\
\text { good or bad? } \\
(0.082)\end{array}$ & $\begin{array}{c}\text { Important in } \\
\text { children: hard work } \\
(0.139)\end{array}$ & $\begin{array}{l}\text { Important in } \\
\text { children: thrift }(0.13)\end{array}$ & $\begin{array}{c}\text { Justifiability of } \\
\text { euthanasia }(0.166)\end{array}$ \\
\hline 7 & $\begin{array}{c}\text { Financial } \\
\text { satisfaction }(0.132)\end{array}$ & $\begin{array}{l}\text { Less importance on } \\
\text { work in future: good } \\
\quad \text { or bad? }(0.249)\end{array}$ & $\begin{array}{l}\text { Justifiability of } \\
\text { divorce }(0.094)\end{array}$ & $\begin{array}{l}\text { Important in } \\
\text { children: religious } \\
\text { faith }(0.081)\end{array}$ & $\begin{array}{l}\text { Important in } \\
\text { children: } \\
\text { unselfishness } \\
(0.138)\end{array}$ & $\begin{array}{l}\text { Important in } \\
\text { children: } \\
\text { unselfishness } \\
(0.129)\end{array}$ & $\begin{array}{c}\text { Financial } \\
\text { satisfaction }(0.163)\end{array}$ \\
\hline 8 & $\begin{array}{l}\text { Political orientation } \\
\qquad(0.13)\end{array}$ & $\begin{array}{l}\text { Confidence in the } \\
\text { police }(0.243)\end{array}$ & $\begin{array}{c}\text { Important in } \\
\text { children: obedience } \\
(0.086)\end{array}$ & $\begin{array}{c}\text { Important in } \\
\text { children: hard work } \\
(0.079)\end{array}$ & $\begin{array}{l}\text { Important in } \\
\text { children: } \\
\text { determination and } \\
\text { perseverance } \\
(0.137)\end{array}$ & $\begin{array}{c}\text { Important in } \\
\text { children: hard work } \\
(0.127)\end{array}$ & $\begin{array}{l}\text { Confidence in the } \\
\text { parliament }(0.162)\end{array}$ \\
\hline 9 & $\begin{array}{c}\text { Important in } \\
\text { children: obedience } \\
(0.121)\end{array}$ & $\begin{array}{l}\text { Importance of God } \\
\qquad(0.242)\end{array}$ & $\begin{array}{l}\text { Life satisfaction } \\
(0.086)\end{array}$ & $\begin{array}{c}\text { Important in } \\
\text { children: tolerance } \\
(0.077)\end{array}$ & $\begin{array}{c}\text { Justifiability of } \\
\text { homosexuality } \\
(0.125)\end{array}$ & $\begin{array}{l}\text { Important in } \\
\text { children: religious } \\
\text { faith }(0.12)\end{array}$ & $\begin{array}{c}\text { Justifiability of } \\
\text { cheating on taxes } \\
(0.154)\end{array}$ \\
\hline 10 & $\begin{array}{c}\text { Political action: } \\
\text { Signing a petition } \\
(0.121)\end{array}$ & $\begin{array}{l}\text { Political action: } \\
\text { Signing a petition } \\
(0.24)\end{array}$ & $\begin{array}{c}\text { Important in } \\
\text { children: hard work } \\
(0.084)\end{array}$ & $\begin{array}{l}\text { Important in } \\
\text { children: feeling of } \\
\text { responsibility } \\
(0.077)\end{array}$ & $\begin{array}{l}\text { Importance of God } \\
\qquad(0.12)\end{array}$ & $\begin{array}{c}\text { Important in } \\
\text { children: } \\
\text { determination and } \\
\text { perseverance } \\
(0.117)\end{array}$ & $\begin{array}{l}\text { Attend religious } \\
\text { services }(0.147)\end{array}$ \\
\hline
\end{tabular}

Note. Numbers in parentheses refer to the dropout loss associated with each predictor within that column—-the bigger the number, the more important the variable. 
Consistent with the secularization hypothesis (Bruce, 2002, 2011), there is a trend toward lower religiosity over time (see Figure 3).

Social attitudes. The second most important set of predictors that helped distinguish respondents from all seven waves referred to social attitudes. The justifiability of homosexuality (variable f118) and abortion (variable f120) helped distinguish respondents across all seven waves. Several related constructs helped distinguish people from different pairs of waves: the justifiability of divorce (variable f121) and euthanasia (variable f122), and the importance of tolerance (variable a035; see Figure 4). Of these, the acceptance of homosexuality showed the sharpest increase over successive years, consistent with past research (e.g., Loftus, 2001). The overall trend is toward greater liberalization of attitudes in these domains (Inglehart \& Baker, 2000).

Independence. The next most important predictor that helped distinguish respondents from all seven waves was the importance of independence in children (variable a029; see Figure 5). The ninth most important predictor assessed the importance of obedience in children (variable a042). Additionally, a variable assessing whether greater respect for authority in the near future would be a bad thing (variable e018) distinguished respondents from pairs of waves. These findings are consistent with the existing literature documenting consistent increases in individualism over time (Santos et al., 2017).

Protestant work ethic / Confucian work ethic. The next most important predictor that helped distinguish respondents from all seven waves was the importance of hard work in children (variable a030). This variable consistently increased from waves 1 to 4 , and then declined slightly from waves 4 to 7 (see Figure 6). On a related note, the importance of thrift (variable a038), determination (variable a039), and responsibility (variable a032) in children contributed to distinguishing respondents between various pairs of waves. These constructs appear closely related to the Protestant work ethic (Weber, 1930) and the Confucian work ethic (Lim, 2003), both of which emphasize hard work, personal responsibility, and thrift. A related 
construct, whether less importance on work in the future would be a bad thing (variable e015), helped distinguish respondents from waves 1 vs. 2 and 2 vs. 3 . This variable gradually decreased from wave 1 to wave 7, likely indicating an increased emphasis on leisure, and thus diverged from the item emphasizing hard work in children.

Life satisfaction. The next most important predictor that helped distinguish respondents from all seven waves was financial satisfaction (variable c006). A related variable, life satisfaction (variable a170), helped distinguish people between wave 2 and 3 . Both these variables declined from waves 1 to 3 , and then gradually increased (see Figure 3 ).

Political attitudes. Next, political orientation (variable e033) helped distinguish respondents from all seven waves (see Figure 7). A related predictor referred to participants' tendency to engage in political action, that is, whether they have ever signed a petition, might do so in the future, or would never do so (variable e025). Respondents' political orientation became more liberal between waves 1 and 2, and somewhat more liberal between waves 6 and 7 . Respondents' proclivity to sign a petition increased from wave 1 and 2 , then gradually declined until wave 6 , and then increased once again. Additional political attitudes helped distinguish respondents between various pairs of waves: confidence in the police (variable e069_06; waves 1 vs. 2), and confidence in the armed forces and the parliament (variables e069_02 and e069_07; waves 6 and 7).

Prosociality. Three questions related to prosociality were relevant in distinguishing respondents between different pairs of waves, including the importance of unselfishness in children (variable a041), and the justifiability of accepting bribes (variable f117) and of cheating on taxes (variable f116). The importance of unselfishness increased from wave 1 to wave 6 , but decreased from wave 6 to wave 7 (see Figure 5). The justifiability of claiming unauthorized government benefits and the justifiability of accepting bribes increased gradually from waves 4 to 7 . These findings indicate a curvilinear pattern, such that the importance of unselfishness reduced in recent years, whereas the justifiability of fraud increased. 
Figure 3

Changes in religiosity-related variables over time. Frequency of attending religious services was reverse-scored-higher numbers indicate greater frequency of attending religious services.

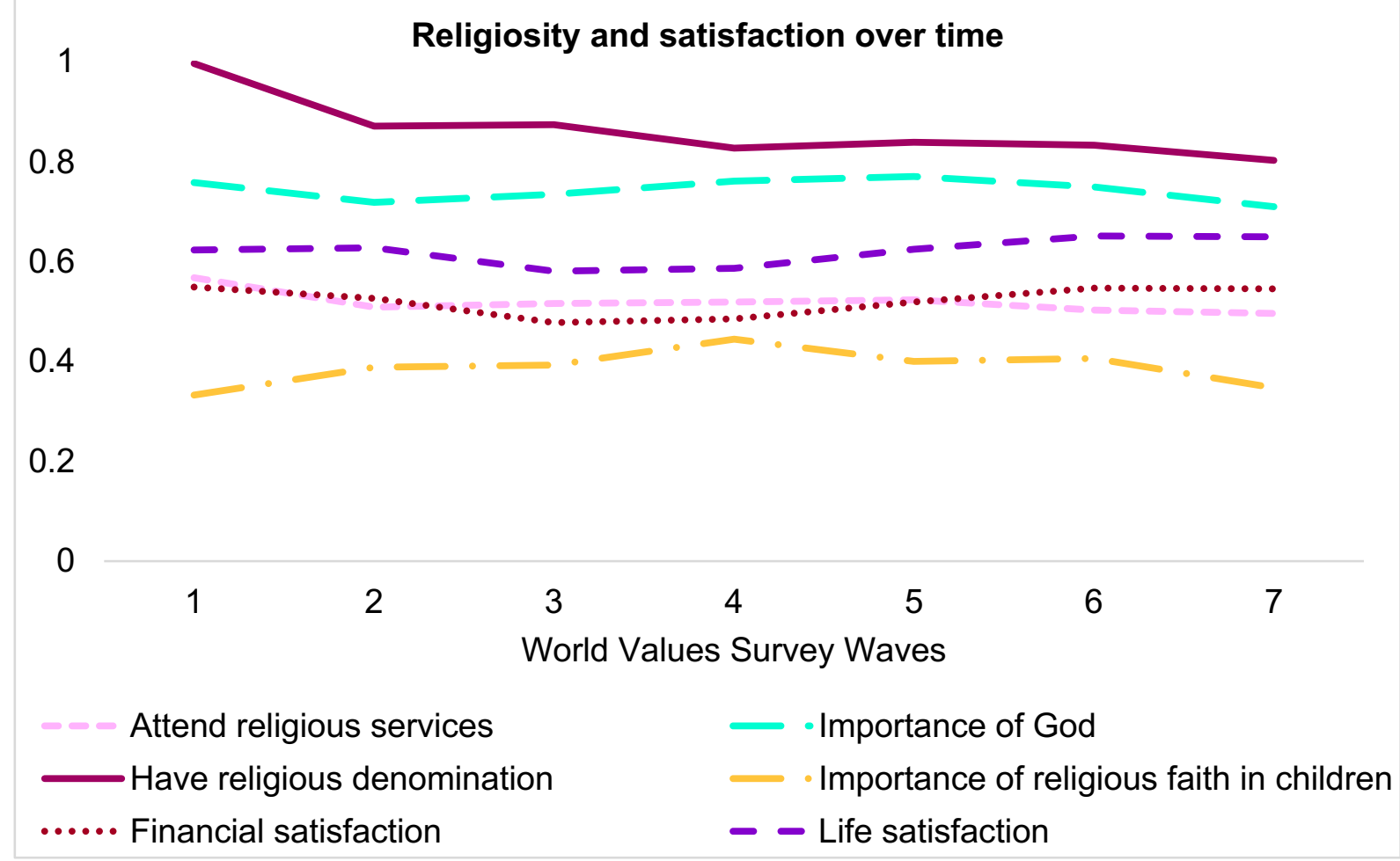

\section{Figure 4}

Changes in social attitudes over time.

Social attitudes over time

1

0.8

0.6

0.4

0.2

0

$$
\begin{array}{lllllll}
1 & 2 & 3 & 4 & 5 & 6 & 7 \\
& \text { World Values Survey Waves } & & &
\end{array}
$$

- Homosexuality justifiable

- Abortion justifiable

- Divorce justifiable

- Euthanasia justifiable

- Importance of tolerance in children 
Figure 5

Changes in independence and prosociality over time.

0.8

Independence and prosociality over time

0.6

0.4

0.2

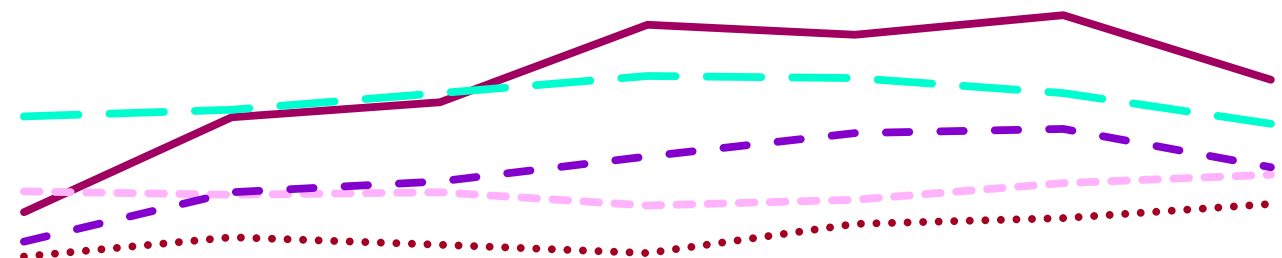

0

1

2

4

5

6

7

World Values Survey Waves

Importance of independence in children

- Importance of obedience in children

- - Greater respect for authority in future is a bad thing

- Importance of unselfishness in children

...... Justifiable to claim government benefits to which one is not entitled

- Justifiable to accept bribes

\section{Figure 6}

Changes in the Protestant work ethic over time.

1

Protestant work ethic over time

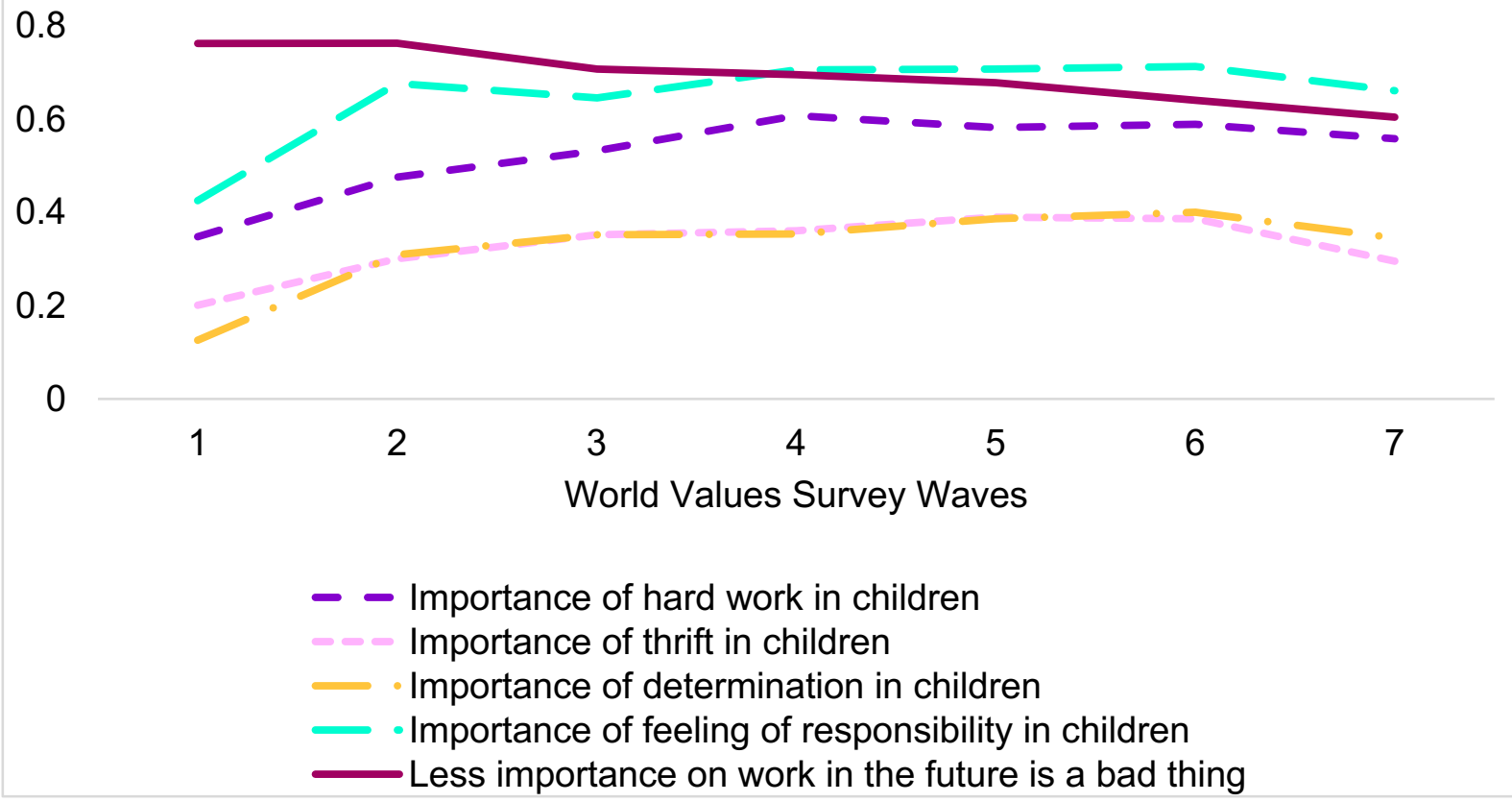




\section{Figure 7}

Changes in political attitudes over time. Signing a petition, confidence in police, and confidence in parliament have been reverse-scored so that higher numbers indicate greater willingness to sign a petition / more confidence in the police / the parliament / the armed forces.

\section{Political attitudes over time}

\section{7}

0.6

0.5

0.4

0.3

0.2

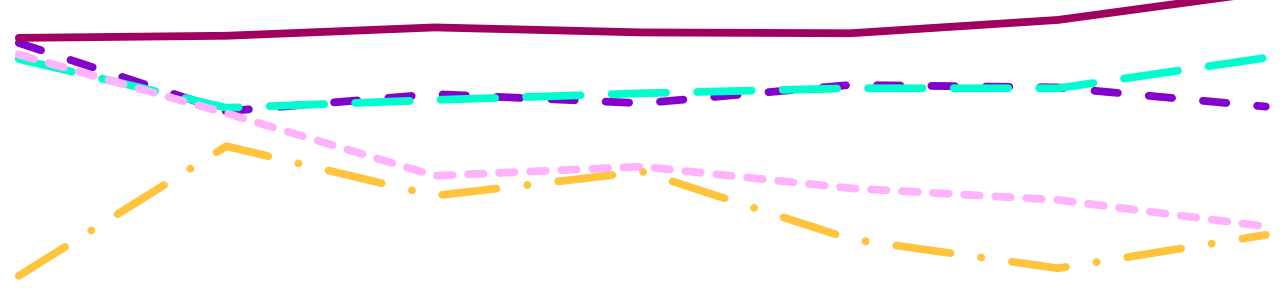

$$
\begin{array}{ccc}
2 & 4 & 5 \\
\text { World Values Survey Waves } \\
- \\
- \text { - Political orientation (higher }=\text { more right) } \\
- \text { Signing a petition } \\
- \text { - Confidence in police } \\
-- \text { Confidence in parliament } \\
\text { - Confidence in armed forces }
\end{array}
$$

\section{Patterns of cultural change within different sub-regions}

Next, we analyzed the variables that contributed the most to distinguishing respondents from different waves within each ISO sub-region (from model 2; see file Top_predictors_model2.xlsx on the OSF repository). Note that not all sub-regions were sampled in all waves. In Northern America, the key variables that distinguished respondents from different waves were religiosity, ethicality, attitudes toward homosexuality, political attitudes, and Protestant work ethic. Life satisfaction and independence, which featured in the global model 1, did not contribute to distinguishing North Americans from different waves. In Latin America and the Caribbean, the findings were fairly similar except that independence also contributed to distinguishing respondents from different waves.

In Northern Europe (i.e., the UK, the Nordic countries, and the Baltic states), the key features were Protestant work ethic, religiosity, social attitudes, independence, political 
attitudes, and a distinctive factor-national pride. An examination of the means across waves indicated that national pride increased from wave 1 to wave 5 , but then decreased in wave 6 . The findings from Western Europe (i.e., French, German, and Dutch speaking countries) were fairly similar. National pride once again featured among the top 10 variables. It decreased from wave 2 to wave 3, but then gradually increased till wave 7. In Southern Europe (i.e., Portugal, the Mediterranean countries, countries in the former Yugoslavia) and Eastern Europe (i.e., the remaining former Communist countries), religiosity, social attitudes, political attitudes, financial satisfaction, and independence contributed the most to distinguishing people across different waves.

In Northern Africa (i.e., countries on the Mediterranean coast, Sudan, and Western Sahara), religiosity, political attitudes, and Protestant work ethic contributed the most to distinguishing people across different waves. Importantly, health status featured as a key variable-it improved from waves 4 to 6 , but then declined in wave 7 . Independence, social attitudes, and life satisfaction did not contribute as much in Northern Africa, and prosociality did not feature in either Northern or Sub-Saharan Africa. In Sub-Saharan Africa, religiosity, political orientation, life satisfaction, and social attitudes were relevant. However, unlike the Americas and Europe, half of the top 10 predictors in Sub-Saharan Africa dealt with characteristics that are important for children. They covered the themes of Protestant work ethic and independence that featured in the global model. The importance of hard work, independence, responsibility, obedience, and thrift all increased from wave 1 to wave 5 or 6 , and then declined in the last one or two waves. It thus appears that people's attitudes about childrearing were particularly relevant for distinguishing people across different waves in Sub-Saharan Africa.

In Western Asia (i.e., Turkey, and countries in the Caucasus and the Arabian peninsula), religiosity, social attitudes, financial satisfaction, political attitudes, Protestant work ethic, and respect for authority contributed the most to distinguishing people across different waves. In Southern Asia (i.e., Iran and the Indian sub-continent), religiosity, political attitudes, social 
attitudes, importance of obedience, and freedom of choice and control contributed the most to distinguishing respondents across different waves. Life satisfaction and prosociality did not feature. This was the only sub-region in which the item assessing freedom of choice and control helped distinguish respondents across different waves. This variable decreased from waves 2-3 to wave 4 , but then increased sharply till wave 7 . Thus, in recent decades, South Asians are experiencing more freedom of choice and control, which is associated with independence (Savani et al., 2008).

In South-eastern Asia (i.e., the region east of the Indian sub-continent and south of China), the key predictors were religiosity, Protestant work ethic, political attitudes, independence, and tolerance. Eastern Asia (i.e., China, Japan, Korea, Mongolia, and Taiwan) was one of the only two sub-regions in which religiosity was not the topmost factor that differentiated respondents across different waves. Instead, the most important factor was whether greater respect for authority would be a bad thing. This variable is on a downward trend over the years, indicating reducing respect for authority over time. A variable unique to Eastern Asia was willingness to fight for the country. This variable increased from waves 1 to 2 , but has been on a decline since. National pride also featured among the top 10 predictors. It increased between waves 1 and 2, then decreased until wave 5, and then increased again in waves 6 and 7. The remaining predictors corresponded to religiosity, political attitudes, social attitudes, and importance of leisure. Australia and New Zealand represented the other sub-region in which religiosity was not the top predictor. Instead, signing a petition, and justifiability of prostitution and homosexuality, contributed the most to distinguishing people across different waves. Other predictors included other political attitudes, religiosity, importance of authority, and emphasis on leisure. Life satisfaction and prosociality did not feature.

\section{Patterns of cultural change within different countries}

In additional analyses, we examined the variables that contributed the most to distinguish different waves within each country (model 3; see file Top_predictors_model3.xlsx 
on the OSF repository). Variables related to religiosity featured among the top 10 predictors for most countries; however, there was a large amount of between-country variability in terms of the variables most helpful in distinguishing respondents from different waves. Given the large number of countries, we do not discuss country-by-country results here. Further, given the paucity of waves in many countries, these results should be interpreted with caution. The rate of missing values varied substantially across the various country-wave combinations (see file MissingValues.xlsx on the OSF repository). Therefore, the accuracy statistics and variance importance results of model 3 need to be interpreted with caution.

\section{Lasso regression}

In further analysis, we ran a multinomial lasso regression (Tibshirani, 1996) in the training data to predict participants' wave based on all 61 variables, using 10-fold crossvalidation to determine the appropriate lambda value. Lasso regressions automatically drop observations with even a single missing value, so this analysis was performed on 122,676 responses without any missing value across the training data and the unseen data. The lasso regression's accuracy in the unseen data was $44.21 \%$ (kappa $=29.62 \%)$, lower than the XGBoost models' accuracy indicated in Table 1. The lasso regression identified the relevant predictors for distinguishing each wave from the baseline wave (i.e., wave 1), but does not have an in-built feature to rank order the predictors in terms of their contribution to simultaneously distinguishing all seven waves. We thus again used the permutation-based DALEX package to rank order the predictors in terms of their contribution to distinguishing all seven waves in the lasso model.

The top 10 predictors were justification of homosexuality, political action: signing a petition, justification of abortion, importance of God, confidence in the parliament, justifiability of claiming undeserved government benefits, importance of obedience and unselfishness in children, and being an atheist (the complete list of variables is available in the Lasso_Predictors.xlsx file on OSF). Most of these variables were also identified as important 
predictors by the XGBoost model; however, the XGBoost model identified variables associated with the Protestant work ethic and life satisfaction, which were not identified by the lasso regression. The partial overlap between the two models is not surprising because the lasso regression makes a number of assumptions (e.g., sparsity; Hastie et al., 2015), whereas the XGBoost model does not make any assumptions (Chen \& Guestrin, 2016).

\section{Variables not contributing to distinguishing respondents from different waves}

Of the 61 variables included in our analysis, only a minority contributed to distinguishing respondents from different waves, both globally (model 1) and within each sub-region (model 2). A key question thus arises: Were the remaining variables largely constant across successive waves, or whether they did change across waves but were not as diagnostic of the waves in which respondents were sampled as the other variables? To answer this question, for each of the 61 variables, we computed the mean for each wave, and then computed the standard deviation of the mean across all waves. Next, we ranked the 61 variables in terms of their standard deviation. This information has been uploaded to the online data repository (see file WVS_variables_used.xlsx).

The top 3 variables with the highest variance across waves (i.e., importance of God, the justifiability of homosexuality, and financial satisfaction) all featured as top predictors in model 1 and model 2. The next two variables in the variance ranking-justifiability of euthanasia and abortion—did not contribute highly to distinguishing all seven waves (model 1), but did contribute to distinguishing specific pairs of waves (model 1), as well as waves within different sub-regions (model 2). Several variables in the top third in terms of the variance ranking, including justifiability of prostitution and suicide, did not feature in either model 1 or model 2. Three variables-importance of independence in children, less importance on work in the future, and greater respect on authority in the future, scored relatively low in the variance ranking (rank 26,31 , and 37 , respectively), but featured among the top 10 variables that distinguished people across waves in model 1 . Thus, the machine learning model was not simply picking up variables 
that varied the most across waves.

\section{Discussion}

In most research on cultural change in cultural psychology, researchers examine how the mean levels of a few theoretically driven variables changed across successive years (e.g., Greenfield, 2013; Grossmann \& Varnum, 2015; Twenge et al., 2012). This is also the case with past research on cultural change using the World Values Survey (e.g., Hamamura, 2012; Inglehart \& Baker, 2000; Santos et al., 2017). In contrast, the machine learning method that we used considers all possible variables as relevant to cultural change, without being restricted by existing theories. This feature leaves open the possibility that the machine learning model can identify variables that are not currently theorized to be important to cultural change. Further, the machine learning model does not solely focus on the mean levels of variables across time several variables that had low variance across waves were used by the machine learning model to identify respondents' wave, whereas a number of variables with high variance were not used. Of the cluster of constructs identified by the machine learning model, four (i.e., religiosity, social attitudes, independence, and satisfaction) have been well-studied in the literature on cultural change, whereas the remaining three (i.e., Protestant work ethic, political attitudes, and prosociality) appear to be relatively less examined in this literature.

The findings about changes in political attitudes are consistent with the general idea that the world is moving toward greater liberalism; however, past research has defined liberalism quite broadly to include a wide range of social attitudes and economic policies (Inglehart \& Baker, 2000; Simmons et al., 2006). To our knowledge, cultural psychologists have not considered political orientation as a key feature of cultural change. We found that globally, people's political orientation is moving from center-right toward center-left, with a marked shift from 2010-2014 to 2017-2019. This finding seems counter to the fact that many Western democracies saw the ascendence of right-wing parties in the second decade of the $21^{\text {st }}$ century. Perhaps part of the increase in liberalism might be a reaction to this ascendance. Visible 
examples of political activism in recent years, such as the 2020 Black Lives Movement and the 2021 US Capitol riots, are consistent with the machine learning model's suggestion people's tendency to engage in political action has been increasing. The 2008 global financial crisis and the increased level of income inequality worldwide (Piketty, 2014) might have precipitated the trend toward more left-wing political ideologies and increased political action in the most recent decade. Given the economic havoc caused by the Covid-19 pandemic in 2020-2021, we would expect these trends to persist in the coming decade. Overall, these findings suggest that psychologists can fruitfully examine changes in political orientation and attitudes.

Although there is a substantial literature on the Protestant work ethic (Furnham, 1984; Weber, 1930) and the Confucian work ethic (Lim, 2003), and although researchers have emphasized a key role of the Protestant work ethic during the reformation period and before (Andersen et al., 2017), to our knowledge, researchers studying cultural change have not considered the Protestant work ethic as a key construct varying over recent decades. The findings indicate that the importance of values related to the Protestant work ethic had been increasing worldwide until the early $21^{\text {st }}$ century but have reached their peak and have begun declining in recent years. A potential explanation is that hard work has been a key means for economic mobility in most countries over most of the past four decades (e.g., Ali, 1992; Ferreira et al., 2012). However, economic mobility has declined in recent years (Katz \& Krueger, 2017), which can explain the reduction in the importance of the Protestant work ethic in the most recent $7^{\text {th }}$ wave of the World Values Survey (2017-2019).

Prosociality is a major topic of research in psychology (e.g., Penner et al., 2005), sociology (e.g., Simpson \& Willer, 2015), and economics (e.g., Benabou \& Tirole, 2006). Although extensive research has documented cultural similarities (e.g., Klein et al., 2015) and differences (Strombach et al., 2013) in generosity, to our knowledge, researchers have not systematically studied changes in prosociality over time. Our findings indicate a curvilinear pattern of change over time. The justifiability of accepting bribes and claiming unauthorized 
government benefits reached a floor around 1999-2004 and then started increasing. The importance of unselfishness in children gradually increased over time but declined in the most recent wave of the WVS. A similar curvilinear pattern is visible in the Gallup ${ }^{\mathrm{TM}}$ World Giving Index survey (Charities Aid Foundation, 2019), which asked respondents across a large number of countries to indicate whether they had donated to charity, volunteered their time, or helped a stranger in the past month. The mean level of the index across the world generally increased from 2010 to 2016 but has declined since. Perhaps increasing income inequality over recent years can explain this pattern (Côté et al., 2015). Future research can investigate this and other possible explanations for this curvilinear pattern.

Past research on cultural change has primarily focused on psychological variables that are changing similarly across the globe. The machine learning model identified many such variables, as discussed above, but it also identified culture-specific markers of cultural change. Religiosity was the dominant factor across the globe except for Eastern Asia, where reduced respect for authority was the most important marker of cultural change, and in Australia and New Zealand, where political action was the most important marker. National pride was relevant to cultural change in Northern Europe, Western Europe, and Eastern Asia; freedom of choice and control was particularly relevant in Southern Asia; and importance of hard work, independence, responsibility, and thrift in children in Sub-Saharan Africa. These findings suggest that researchers can fruitfully examine both culture-general and culture-specific aspects of cultural change.

In addition to identifying which psychological factors were key markers of cultural change, the present analysis identified variables that were not. In particular, generalized trust (variable a165), a key variable in sociology, economics, psychology, and political science, including in past research on cultural change (e.g., Hamamura, 2012), did not feature among the top 10 variables in either the global model or the sub-region model. Neither did racism, that is, whether respondents would be comfortable with a person of a different race as a neighbor 
(variable a124_02), despite likely decreases in explicit racism over the years (Swim et al., 1995). And neither did xenophobia, that is, whether respondents would be comfortable with a foreigner as a neighbor (variable a124_06). Although people's health and possibly their happiness increased over the years with economic development and poverty reduction, people's self-related health status and happiness were not among the key markers of cultural change (except in Northern Africa). In contrast, their life satisfaction and financial satisfaction were important markers of cultural change.

Understandably, the WVS researchers designed the survey to measure a large number of different constructs to maximize the breadth of coverage, rather than measuring a narrower set of constructs using a large number of items that can be used to form a scale. Therefore, we had to analyze the data at the level of individual items rather than at the level of scale averages. Although most of our discussion focused on groups of thematically related items, it would be ideal if the current findings can be verified with external datasets that did measure the relevant constructs using multi-item scales over multiple decades in multiple countries. Till then, any generalization from individual items to theoretical constructs must be viewed as tentative.

The WVS sampled different countries in different waves. This inconsistency raises the concern that the changes in values across different periods are confounded with the sampling of different countries in different periods. This is a shortcoming of the global model 1. However, it is less of a concern in model 2, which assesses changes in values within each sub-region, as countries within a sub-region tend to be culturally similar (Inglehart \& Baker, 2000; Schwartz, 2006). To address this shortcoming, when analyzing the results of model 1 , we plotted the change in values over successive waves while accounting country effects. The results depicted in the graphs can thus be interpreted as the expected change in values assuming that the same countries were sampled across all waves.

A key limitation of machine learning methods is that they inherit the limitations of existing large datasets (Zou \& Schiebinger, 2018). There exist sufficiently large social science datasets, 
such as the World Values Survey and the General Social Survey, on which machine learning analyses can be performed. However, such datasets exclusively contain individuals' responses to a large number of self-report questions. Although much can be gleaned from self-reports, self-reports have limited utility in cultural psychology (Heine et al., 2002). Much of the advancements in the field has come from the insight that culture is represented in patterns of cognition, emotion, and motivation that people might not have conscious access to (Adams \& Markus, 2004; Kitayama, 2002), but which can be measured using behavioral tasks that assess implicit tendencies (Kitayama et al., 2009). However, large enough datasets of implicit tendencies do not exist, and compiling them would entail enormous costs. Thus, as of now, machine learning methods cannot be used to study implicit psychological tendencies.

The method that we used did not automatically group items into thematically related categories. However, psychologists are typically not interested in individual items but in the underlying constructs. Machine learning methods for dimensionality reduction exist, but in supervised learning (in which the model is trying to predict an outcome variable), the latent dimensions are typically constructed to accurately predict the outcome variable, not to represent coherent constructs (van der Maaten et al., 2009). Given that psychologists prioritize cogent explanations over predictive accuracy (Yarkoni \& Westfall, 2017), less than ideal interpretability is a limitation of machine learning models. Unsupervised machine learning methods can be used for dimensionality reduction without prioritizing prediction, but once again, there is no requirement that the items feeding into the latent dimensions cohere together.

In conclusion, the machine learning approach to cultural change verified the significance of changes in independence, which has been a critical focus of this literature. The machine model's findings about the significance of religiosity and social attitudes are consistent with the sociological literature on cultural change. However, the machine learning approach also identified multiple other aspects of cultural change that have not received much attention, including Protestant work ethic, political orientation, political action, and prosociality. Thus, the 
current research indicates that theory-blind machine learning approaches can complement traditional theory-driven approaches to generate novel insights that researchers might miss otherwise (Bleidorn \& Hopwood, 2019; Sheetal et al., 2020). 


\section{References}

Adams, G., \& Markus, H. R. (2004). Toward a conception of culture suitable for a social psychology of culture. In M. Schaller \& C. S. Crandall (Eds.), The psychological foundations of culture (pp. 335-360). Erlbaum.

Ali, A. J. (1992). The Islamic work ethic in Arabia. The Journal of Psychology, 126(5), 507-519.

Altmann, A., Toloşi, L., Sander, O., \& Lengauer, T. (2010). Permutation importance: a corrected feature importance measure. Bioinformatics, 26(10), 1340-1347.

Andersen, T. B., Bentzen, J., Dalgaard, C. J., \& Sharp, P. (2017). Pre-reformation roots of the protestant ethic. The Economic Journal, 127(604), 1756-1793.

Bénabou, R., \& Tirole, J. (2006). Incentives and prosocial behavior. American Economic Review, 96(5), 1652-1678.

Biecek, P. (2018). Dalex: Explainers for complex predictive models in r. Journal of Machine Learning Research, 19, 1-5.

Bleidorn, W., \& Hopwood, C. J. (2019). Using machine learning to advance personality assessment and theory. Personality and Social Psychology Review, 23(2), 190-203.

Breiman, L. (2001). Random forests. Machine Learning, 45(1), 5-32.

Breiman, L., Friedman, J., Stone, C. J., \& Olshen, R. A. (1984). Classification and regression trees. CRC press.

Bruce, S. (2002). God is dead: Secularization in the West (Vol. 3). Blackwell.

Bruce, S. (2011). Secularization: In defense of an unfashionable theory. Oxford University Press.

Charities Aid Foundation (2019). CAF World Giving Index 10th edition. https://www.cafonline.org/about-us/publications/2019-publications/caf-world-givingindex-10th-edition

Chen, T., \& Guestrin, C. (2016). XGBoost: A scalable tree boosting system. Proceedings of the 22nd ACM SIGKDD international conference on knowledge discovery and data mining, 16, 785-794. https://doi.org/10.1145/2939672.2939785

Cohen, D. (2001). Cultural variation: considerations and implications. Psychological Bulletin, 127(4), 451-471. 
Côté, S., House, J., \& Willer, R. (2015). High economic inequality leads higher-income individuals to be less generous. Proceedings of the National Academy of Sciences, 112(52), 15838-15843.

Englebert, P., Tarango, S., \& Carter, M. (2002). Dismemberment and suffocation: A contribution to the debate on African boundaries. Comparative Political Studies, 35(10), 1093-1118.

ErcegHurn, D. M., \& Mirosevich, V. M. (2008). Modern robust statistical methods: An easy way to maximize the accuracy and power of your research. American Psychologist, 63, 591-601.

Ferreira, F. H., Messina, J., Rigolini, J., López-Calva, L. F., Lugo, M. A., Vakis, R., \& Ló, L. F. (2012). Economic mobility and the rise of the Latin American middle class. World Bank Publications.

Furnham, A. (1984). The protestant work ethic: A review of the psychological literature. European Journal of Social Psychology, 14, 87-104.

Gómez-Ramírez, J., Ávila-Villanueva, M., \& Fernández-Blázquez, M. Á. (2020). Selecting the most important self-assessed features for predicting conversion to mild cognitive impairment with random forest and permutation-based methods. Scientific Reports, 10(1), 1-15.

Goretzko, D., \& Bühner, M. (2020). One model to rule them all? Using machine learning algorithms to determine the number of factors in exploratory factor analysis. Psychological Methods, 25(6), 776-786.

Greenfield, P. M. (2013). The changing psychology of culture from 1800 through 2000. Psychological Science, 24, 1722-1731.

Greenfield, P. M. (2016). Social change, cultural evolution, and human development. Current Opinion in Psychology, 8, 84-92.

Grossmann, I., \& Varnum, M. E. (2015). Social structure, infectious diseases, disasters, secularism, and cultural change in America. Psychological Science, 26, 311-324.

Guo, X., Yin, Y., Dong, C., Yang, G., \& Zhou, G. (2008, October). On the class imbalance problem. In 2008 Fourth International Conference on Natural Computation (Vol. 4, pp. 192-201). IEEE. 
Haerpfer, C., Inglehart, R., Moreno, A., Welzel, C., Kizilova, K., Diez-Medrano J., M. Lagos, P. Norris, E. Ponarin \& B. Puranen et al. (2020). World Values Survey: Round Seven - Country-Pooled Datafile. Madrid, Spain \& Vienna, Austria: JD Systems Institute \& WVSA Secretariat. https://doi.org/10.14281/18241.1

Hamamura, T. (2012). Are cultures becoming individualistic? A crosstemporal comparison of individualism-collectivism in the United States and Japan. Personality and Social Psychology Review, 16, 3-24.

Hastie, T., Tibshirani, R., \& Wainwright, M. (2015). Statistical learning with sparsity: The lasso and generalizations. CRC press.

Heine, S. J., Lehman, D. R., Peng, K., \& Greenholtz, J. (2002). What's wrong with crosscultural comparisons of subjective Likert scales? The reference-group effect. Journal of Personality and Social Psychology, 82, 903-918.

Huang, J., \& Ling, C. X. (2005). Using AUC and accuracy in evaluating learning algorithms. IEEE Transactions on knowledge and Data Engineering, 17(3), 299-310.

Inglehart, R., \& Baker, W. E. (2000). Modernization, cultural change, and the persistence of traditional values. American Sociological Review, 65, 19-51.

ISO (International Organization for Standardization). (2006). ISO 3166-1: Codes for the representation of names of countries and their subdivisions-Part 1: Country codes.

Katz, L. F., \& Krueger, A. B. (2017). Documenting decline in US economic mobility. Science, 356(6336), 382-383.

Kitayama, S. (2002). Culture and basic psychological processes-Toward a system view of culture: Comment on Oyserman et al. (2002). Psychological Bulletin, 128, 89-96.

Kitayama, S., Park, H., Sevincer, A. T., Karasawa, M., \& Uskul, A. (2009). A cultural task analysis of implicit independence: Comparing North America, Western Europe, and East Asia. Journal of Personality and Social Psychology, 97, 236-255.

Klein, N., Grossman, I., Uskul, A. K., Kraus, A., \& Epley, N. (2015). It pays to be nice, but not really nice: Asymmetric evaluations of prosociality across seven cultures. Judgment and Decision Making, 10, 355-364.

Kleinberg, J., Lakkaraju, H., Leskovec, J., Ludwig, J., \& Mullainathan, S. (2018). Human decisions and machine predictions. The Quarterly Journal of Economics, 133(1), 237- 
293.

Landis, J. R., \& Koch, G. G. (1977) The measurement of observer agreement for categorical data. Biometrics, 33(1), 159-174.

Lim, V. K. (2003). Money matters: An empirical investigation of money, face and Confucian work ethic. Personality and Individual Differences, 35(4), 953-970.

Loftus, J. (2001). America's liberalization in attitudes toward homosexuality, 1973 to 1998. American Sociological Review, 66(5), 762-782.

Martinez-Cantin, R. (2014). BayesOpt: a Bayesian optimization library for non-linear optimization, experimental design and bandits. Journal of Machine Learning Research, 15(1), 3735-3739.

Oishi, S., \& Graham, J. (2010). Social ecology: Lost and found in psychological science. Perspectives on Psychological Science, 5(4), 356-377.

Penner, L. A., Dovidio, J. F., Piliavin, J. A., \& Schroeder, D. A. (2005). Prosocial behavior: Multilevel perspectives. Annual Review of Psychology, 56, 365-392.

Piketty, T. (2014). Capital in the Twenty-First Century. The Belknap Press of Harvard University Press.

Rish, I. (2001). An empirical study of the naive Bayes classifier. IJCAI 2001 Workshop on Empirical methods in Artificial Intelligence, 3(22), 41-46. https://www.cc.gatech.edu/ isbell/reading/papers/Rish.pdf

Santos, H. C., Varnum, M. E., \& Grossmann, I. (2017). Global increases in individualism. Psychological Science, 28, 1228-1239.

Savani, K., Markus, H. R., \& Conner, A. L. (2008). Let your preference be your guide? Preferences and choices are more tightly linked for North Americans than for Indians. Journal of Personality and Social Psychology, 95(4), 861-876.

Schwartz, S. (2006). A theory of cultural value orientations: Explication and applications. Comparative Sociology, 5(2-3), 137-182.

Sheetal, A., Feng, Z., \& Savani, K. (2020). Using machine learning to generate novel hypotheses: Increasing optimism about covid19 makes people less willing to justify unethical behaviors. Psychological Science, 31(10), 1222-1235.

Simmons, B. A., Dobbin, F., \& Garrett, G. (2006). Introduction: The international diffusion of 
liberalism. International Organization, 781-810.

Simpson, B., \& Willer, R. (2015). Beyond altruism: Sociological foundations of cooperation and prosocial behavior. Annual Review of Sociology, 41, 43-63.

Strigl, D., Kofler, K., \& Podlipnig, S. (2010, February). Performance and scalability of GPUbased convolutional neural networks. 2010 18th IEEE Euromicro Conference on Parallel, Distributed and Network-based Processing, 317-324.

Strombach, T., Jin, J., Weber, B., Kenning, P., Shen, Q., Ma, Q., \& Kalenscher, T. (2014). Charity begins at home: Cultural differences in social discounting and generosity. Journal of Behavioral Decision Making, 27(3), 235-245.

Swim, J. K., Aikin, K. J., Hall, W. S., \& Hunter, B. A. (1995). Sexism and racism: Old-fashioned and modern prejudices. Journal of Personality and Social Psychology, 68(2), 199-214.

Tibshirani, R. (1996). Regression shrinkage and selection via the lasso. Journal of the Royal Statistical Society: Series B (Methodological), 58(1), 267-288.

Twenge, J. M., Campbell, W. K., \& Gentile, B. (2012). Increases in individualistic words and phrases in American books, 1960-2008. PloS One, 7, e40181.

Twenge, J. M., Campbell, W. K., \& Gentile, B. (2013). Changes in pronoun use in American books and the rise of individualism, 19602008. Journal of CrossCultural Psychology, 44, 406-415.

Van Der Maaten, L., Postma, E. \& Van den Herik, J. (2009). Dimensionality reduction: a comparative review. Journal of Machine Learning Research, 10, 66-71.

Varnum, M. E., \& Grossmann, I. (2016). Pathogen prevalence is associated with cultural changes in gender equality. Nature Human Behaviour, 1, 1-4.

Varnum, M. E., \& Grossmann, I. (2017). Cultural change: The how and the why. Perspectives on Psychological Science, 12, 956-972.

Weber, M. (1930). The Protestant ethic and the spirit of capitalism. Routledge.

Yarkoni, T., \& Westfall, J. (2017). Choosing prediction over explanation in psychology: Lessons from machine learning. Perspectives on Psychological Science, 12(6), 1100-1122.

XGBoost Developers. (2020a). Random Forests in XGBoost. https://xgboost.readthedocs.io/en/latest/tutorials/rf.html XGBoost Developers. (2020b). Understand your dataset with XGBoost. 
https://xgboost.readthedocs.io/en/latest/R-package/discoverYourData.html

Zhang, Z., \& Sabuncu, M. R. (2018). Generalized cross entropy loss for training deep neural networks with noisy labels. arXiv preprint arXiv:1805.07836.

Zou, J., \& Schiebinger, L. (2018). Al can be sexist and racist - it's time to make it fair. Nature, 559, 324-326. doi: 10.1038/d41586-018-05707-8. 